\title{
Accuracy of polyp localization at colonoscopy
}

Authors

Institutions
Sam A. O'Connor ${ }^{1,2}$, David G. Hewett ${ }^{2,3}$, Marcus O. Watson ${ }^{2,4}$, Bradley J. Kendall ${ }^{1,2}$, Luke F. Hourigan', Gerald Holtmann ${ }^{1,5}$

Institutions are listed at the end of article. submitted 7. March 2016 accepted after revision 7. March 2016

\section{Bibliography}

Dol http://dx.doi.org/

10.1055/s-0042-105864

Published online: 19.5.2016

Endoscopy International Open 2016; 04: E642-E646

(c) Georg Thieme Verlag KG Stuttgart · New York

E-ISSN 2196-9736

\section{Corresponding author}

Sam A O'Connor, MBBS (Hons), FRACP

Department of

Gastroenterology

Princess Alexandra Hospital

199 Ipswich Rd

Woolloongabba, Queensland

Australia

Phone: +61-414-447-725

Phone: +61 7-3176-2613

Fax: +61-7-3176-5111

oconnorsama@gmail.com
Background and study aims: Accurate documentation of lesion localization at the time of colonoscopic polypectomy is important for future surveillance, management of complications such as delayed bleeding, and for guiding surgical resection. We aimed to assess the accuracy of endoscopic localization of polyps during colonoscopy and examine variables that may influence this accuracy.

Patients and methods: We conducted a prospective observational study in consecutive patients presenting for elective, outpatient colonoscopy. All procedures were performed by Australian certified colonoscopists. The endoscopic location of each polyp was reported by the colonoscopist at the time of resection and prospectively recorded. Magnetic endoscope imaging was used to determine polyp location, and colonoscopists were blinded to this image. Three experienced colonoscopists, blinded to the endoscopist's assessment of polyp location, independently scored the magnetic endoscope images to obtain a reference standard for polyp location (Cronbach alpha 0.98). The accuracy of colonoscopist polyp locali-

\section{Introduction}

$\nabla$

Colonoscopic polypectomy reduces mortality from colorectal cancer [1]. Precise lesion localization at the time of polypectomy is important, yet rates of incorrect colonoscopic localization are as high as $34 \%$ [2-8]. Localization is relevant for colonoscopic surveillance following piecemeal endoscopic resection, for subsequent surgical resection of malignant or unresectable polyps, and even for the management of complications such as delayed bleeding. Interval colorectal cancers are known to occur at sites of incomplete polypectomy $[9,10]$. Colonoscopic tattoo is typically used to facilitate subsequent identification, but it is not without risk and may not be visible at the time of surgery $[9,11]$. zation using this reference standard was assessed, and colonoscopist, procedural and patient variables affecting accuracy were evaluated.

Results: A total of 155 patients were enrolled and 282 polyps were resected in 95 patients by 14 colonoscopists. The overall accuracy of polyp localization was $85 \%$ (95\% confidence interval, CI; 60 $96 \%)$. Accuracy varied significantly $(P<0.001)$ by colonic segment: caecum $100 \%$, ascending $77 \%$ (CI;65 - 90), transverse $84 \%(\mathrm{Cl} ; 75$ - 92), descending 56\% (CI;32-81), sigmoid 88\% (CI;79-97), rectum $96 \%(\mathrm{CI} ; 90-101)$. There were significant differences in accuracy between colonoscopists $(P<0.001)$, and colonoscopist experience was a significant independent predictor of accuracy (OR 3.5, $P=0.028$ ) after adjustment for patient and procedural variables.

Conclusions: Accuracy of localization of polyps is imprecise and affected by position within the colon and colonoscopist, including their level of experience. Magnetic endoscope imaging may improve the localization of lesions during colonoscopy.

Magnetic endoscope imaging (ScopeGuide, Olympus Medical Systems Corporation, Japan) is a technology to facilitate instrument localization during colonoscopy. Numerous electromagnetic generator coils positioned throughout the scope transmitted to sensors provide an accurate, realtime image of the colonoscope within space [12]. Ellul et al showed that ScopeGuide was able to correctly localize tumor position $93.75 \%$ of the time in 82 colonic tumors compared to surgical resection and localization [13]. Shah et al showed scope tip accuracy around $90 \%$ compared to air contrast abdominal X-ray when localizing colonoscopic placed clips [14].

In this report, we assessed the accuracy of endoscopic localization of polyps by colonoscopists with magnetic endoscope imaging used as a refer- 

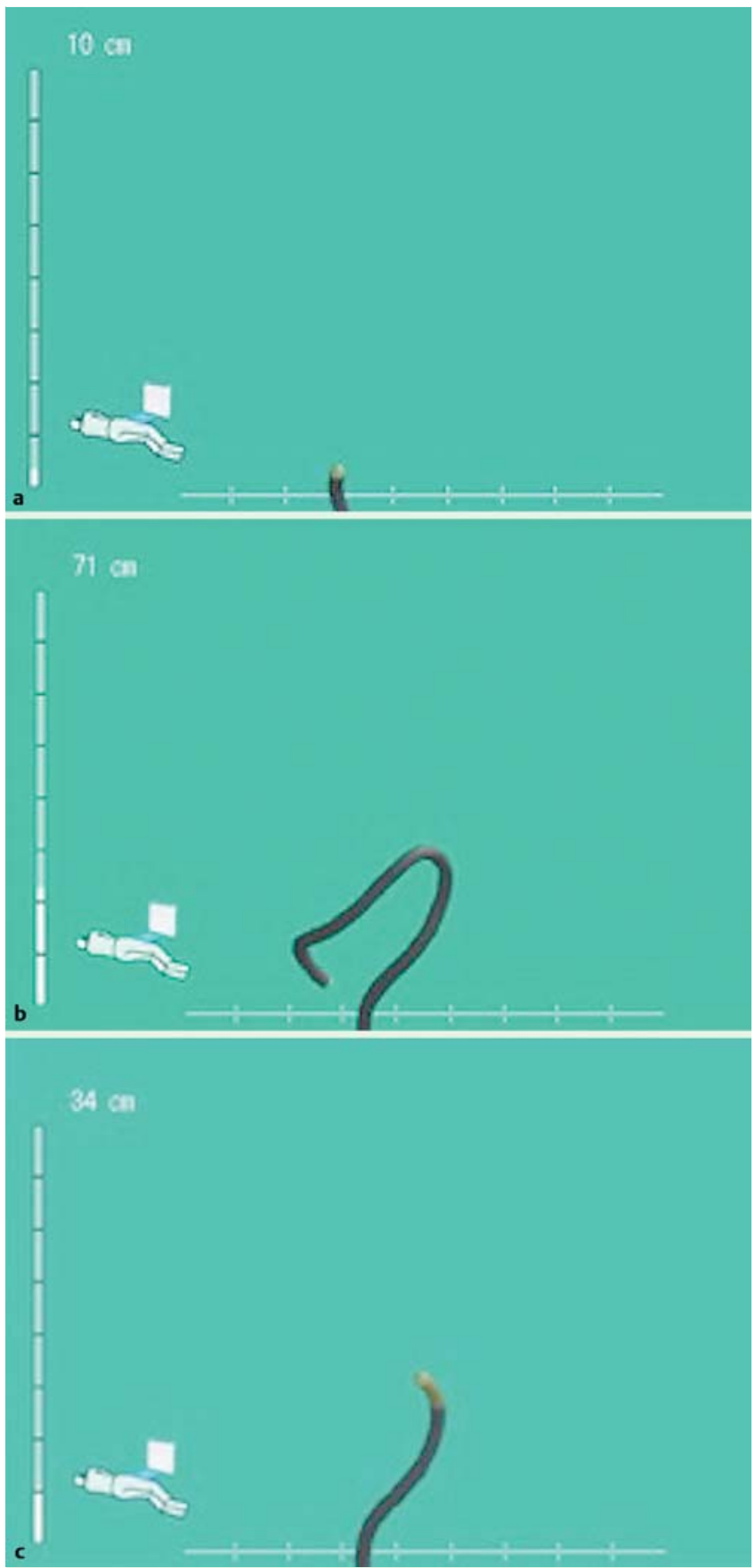

Fig. 1a-c Magnetic endoscope imaging (ScopeGuide) images from patient 63, polyp 119: a rectal intubation; $\mathbf{b}$ cecal intubation; and $\mathbf{c}$ location of polypectomy. The polyp was localized by the colonoscopist at the proximal sigmoid colon, but scored by each experienced colonoscopist using the ScopeGuide image to be at the splenic flexure.

ence standard for polyp location. We also aimed to evaluate accuracy in different segments of the colon and rectum, and examine patient, procedure or colonoscopist factors associated with accuracy of localization.

We hypothesize that the accuracy of colonoscopist localization of the colonoscope tip is variable especially within the mid colon.

\section{Patients and methods}

$\nabla$

\section{Study design/setting}

We conducted a prospective observational study of polyp localization during colonoscopy at an Australian university teaching hospital. The Metro South Health Human Research Ethics Committee approved the study. We followed the "strengthening the reporting of observational studies in epidemiology" (STROBE) guidelines in reporting our findings [15].

\section{Patients and endoscopists}

We enrolled consecutive patients presenting for elective, outpatient colonoscopy. Patients were excluded if they had a history of previous colorectal resection or were unable to give informed consent. Recruitment occurred between 2 September 2013 and 13 November 2013, and patients were enrolled by a research assistant or the authors. Written informed consent was obtained from all patients prior to the procedure.

\section{Endoscopists}

All procedures were performed by qualified endoscopists certified by the Australian Conjoint Committee for Recognition of Training in Gastrointestinal Endoscopy. All participating colonoscopists provided written informed consent prior to commencement of the study.

\section{Procedures}

Colonoscopy was performed using Olympus colonoscopes with magnetic imaging technology (CF-HQ190L, Olympus Medical Systems Corporation, Tokyo, Japan). All data and images were recorded at the time of colonoscopy.

In each patient, colonoscopy was performed to the cecum. During insertion and withdrawal, the magnetic endoscope (ScopeGuide) image was transmitted to a separate room and recorded using a digital video recording device (IMH-20, Olympus Medical Systems Corporation, Tokyo Japan). During instrument insertion, the endoscopist was permitted to view the ScopeGuide image adjacent to the endoscopic image. After cecal intubation, the ScopeGuide image in the endoscopy room was switched off. Polyps removed during instrument insertion were not included. On instrument withdrawal, the endoscopic location of each polyp was determined and reported by the colonoscopist at the time of resection and prospectively recorded by a research assistant in the endoscopy room. A still ScopeGuide image of the instrument location was prospectively obtained on initial insertion to the rectum, on cecal intubation, and at the time of each polypectomy $(\bullet$ Fig. 1 ). These images were captured digitally in real time by the first author in a separate room.

After clinical data collection had concluded, a ScopeGuide image library of all polyps was collated. Three independent experienced colonoscopists (D.G.H., B.J.K, L.F.H.), blinded to the endoscopist's assessment of polyp location, independently reviewed the ScopeGuide images and scored the location of each polyp to obtain a reference standard.

\section{Variables}

Patient variables included age, sex, body mass index (BMI) and colonoscopy indication. Colonoscopist variables included experience and annual colonoscopy volume. For each procedure, we recorded the type of sedation, degree of instrument looping, quality of bowel preparation and procedure time. Degree of looping was reported by each endoscopist using a 4-point scale (none, 
minimal, moderate, substantial). Bowel preparation was assessed using a 4-point scale (excellent, good, fair, poor). For documentation of polyp location by the endoscopist and by the independent raters, the colon and rectum were divided into 18 segments ( $\bullet$ Table 1). These segments were collapsed into 6 anatomic regions for analysis: cecum, ascending colon, transverse colon, descending colon, sigmoid colon, and rectum. The hepatic flexure was included with the ascending colon, the splenic flexure was included with the transverse colon, and the rectosigmoid was included with the sigmoid colon.

The primary outcome was the endoscopist's accuracy of polyp localization. The reference standard for polyp location was determined from the ScopeGuide images, as scored by the 3 experienced endoscopists from 18 segments. For each polyp, if 2 or more segment scores from the experienced endoscopists were concordant, this became the reference standard; if the scores were not concordant, the median segment was used.

Any differences between the colonoscopist localization and the reference standard were scored on the basis of segments deviation (out of 18).

\section{Statistical analysis}

Data and statistical analysis was performed using Stata 12.1 (Statacorp, College Station, TX United States). The accuracy of colonoscopist-reported polyp localization was calculated using the ScopeGuide derived reference standard. For the reference standard, we tested concordance between independent raters using the intraclass correlation coefficient (ICC) and Cronbach alpha. Concordance between experienced colonoscopist ratings of polyp location was very high: ICC 0.99 , Cronbach alpha 0.98 .

A colonoscopist was considered accurate if his or her reported segment was within 2 (out of 18) segments of the ScopeGuide image reference standard. We also assessed the extent of deviation in colonoscopist-reported location from the reference standard, measured in segments and calculated by counting the number of segments of difference between colonoscopic location and reference standard (range 0 to 17). Multiple logistic regression and multiple linear regression analyses were performed to evaluate the impact of patient, procedural, and endoscopist variables on accuracy and on deviation between colonoscopic location and the reference standard.

\section{Results \\ $\nabla$}

A total of 155 patients were recruited ( $\$$ Table 1 ) and 282 polyps were resected in 95 patients by 14 colonoscopists. Five procedures were incomplete due to obstructing cancers $(n=3)$, poor bowel preparation $(n=1)$ and instrument looping $(n=1)$, and 21 polyps were excluded due to failure to capture a ScopeGuide image (leaving 261 polyps for analysis).

Overall accuracy (\% correct) of polyp localization was 85\% (95\% confidence interval: 81.2 to $89.7 \%$ ). Accuracy varied significantly $(P<0.001)$ by colonic segment ( $\bullet$ Table 2, $\bullet$ Fig. 2 ), and was highest in the cecum $(100 \%)$ and lowest in the descending colon (56\%). Of the 38 polyps that were incorrectly localized, 27 (71\%) were localized distal to the ScopeGuide reference standard.

On bivariate analyses, the extent of deviation between actual and predicted location was not significantly influenced by patient age ( $P=0.452)$, sex $(P=0.455)$, BMI $(P=0.186)$ or type of sedation $(P=$ $0.290)$. Similarly, there was no association between accuracy and bowel preparation $(P=0.084)$ or degree of instrument looping
Table 1 Patient characteristics.

\begin{tabular}{|c|c|}
\hline & All patients, $n=155$ \\
\hline \multicolumn{2}{|l|}{ Sex, $n(\%)$} \\
\hline Male & $88(57)$ \\
\hline Female & $67(43)$ \\
\hline \multicolumn{2}{|l|}{ Age, $y$} \\
\hline Mean & 53.9 \\
\hline Median (range) & $55(17-85)$ \\
\hline \multicolumn{2}{|c|}{ Body mass index, $\mathrm{n}(\%)^{1}$} \\
\hline$<20$ & $9(6)$ \\
\hline $20-24$ & $49(32)$ \\
\hline $25-29$ & $63(41)$ \\
\hline $30-34$ & $22(14)$ \\
\hline$\geq 35$ & $10(6)$ \\
\hline \multicolumn{2}{|l|}{ Indication, n (\%) } \\
\hline Screening & $1(1)$ \\
\hline Surveillance & $34(22)$ \\
\hline Symptoms & $120(77)$ \\
\hline \multicolumn{2}{|l|}{ Sedation, n (\%) } \\
\hline Midazolam & $105(68)$ \\
\hline Propofol & $50(32)$ \\
\hline \multicolumn{2}{|c|}{ Instrument looping, $\mathrm{n}(\%)^{2}$} \\
\hline None & $27(21)$ \\
\hline Mild & $64(49)$ \\
\hline Moderate & $21(16)$ \\
\hline Substantial & $18(14)$ \\
\hline
\end{tabular}

'Missing values $=2$

2 Missing values $=25$

$(P=0.405)$. Multiple logistic regression analyses showed no significant association between accuracy and patient or procedural variables.

There was a significant difference in accuracy between colonoscopists $(P<0.001, \otimes$ Table 3$)$. Multiple logistic regression analyses found colonoscopist experience to be a significant independent predictor of accuracy (OR 3.69, $P=0.02$ ), after adjusting for polyp location, patient age, sex, BMI, and type of sedation. Colonoscopist experience was also a significant independent predictor of the extent of deviation between colonoscopic location and reference standard $(P=0.004)$, after adjusting for polyp location age, sex, BMI, and sedation type.

\section{Discussion \\ $\nabla$}

In this study, we have shown that colonoscopic localization of polyps can be imprecise, and is affected by colonic segment and colonoscopist. Accuracy was lowest in the descending colon, followed by the ascending and transverse colon, while colonoscopists were most accurate in the proximal and distal segments of the colorectum. Accuracy varied significantly between colonoscopists and with experience, but was not influenced by patient or procedural variables.

Magnetic endoscope imaging is an established useful adjunct for assisting with loop identification and reduction during colonoscopy, and for reducing patient discomfort in unsedated or minimally sedated colonoscopy [16-19] , although it is less useful for reducing cecal intubation time [20]. However, the role of magnetic endoscope imaging as an adjunctive tool for colonoscopic lesion localization has not be well studied. It has been shown to be highly accurate compared to other imaging modalities and surgical localization $[2-8,14]$. 
Table 2 Location of polyps and accuracy of localization.

\begin{tabular}{|c|c|c|c|c|c|}
\hline Segment & Location & Total polyps & Correct & Incorrect & $\begin{array}{l}\text { Accuracy \% } \\
\text { (95\% confidence interval) }\end{array}$ \\
\hline \multirow[t]{2}{*}{1} & Cecum & 24 & 24 & 0 & \\
\hline & CECUM & 24 & 24 & 0 & 100 \\
\hline 2 & Proximal ascending & 10 & 7 & 3 & \\
\hline 3 & Mid ascending & 15 & 12 & 3 & \\
\hline 4 & Distal ascending & 9 & 5 & 4 & \\
\hline \multirow[t]{2}{*}{5} & Hepatic flexure & 10 & 10 & 0 & \\
\hline & ASCENDING & 44 & 34 & 10 & $77(65-90)$ \\
\hline 6 & Proximal transverse & 25 & 23 & 2 & \\
\hline 7 & Mid transverse & 15 & 13 & 2 & \\
\hline 8 & Distal transverse & 21 & 17 & 4 & \\
\hline \multirow[t]{2}{*}{9} & Splenic flexure & 18 & 13 & 5 & \\
\hline & TRANSVERSE & 79 & 66 & 13 & $84(75-92)$ \\
\hline 10 & Proximal descending & 4 & 3 & 1 & \\
\hline 11 & Mid descending & 4 & 1 & 3 & \\
\hline \multirow[t]{2}{*}{12} & Distal descending & 8 & 5 & 3 & \\
\hline & DESCENDING & 16 & 9 & 7 & $56(32-81)$ \\
\hline 13 & Proximal sigmoid & 12 & 12 & 0 & \\
\hline 14 & Mid sigmoid & 7 & 6 & 1 & \\
\hline 15 & Distal sigmoid & 14 & 12 & 2 & \\
\hline \multirow[t]{2}{*}{16} & Rectosigmoid & 17 & 14 & 3 & \\
\hline & SIGMOID & 50 & 44 & 6 & $88(79-97)$ \\
\hline 17 & Proximal rectum & 28 & 28 & 0 & \\
\hline \multirow[t]{2}{*}{18} & Distal rectum & 20 & 18 & 2 & \\
\hline & RECTUM & 48 & 46 & 2 & $96(90-101)$ \\
\hline
\end{tabular}

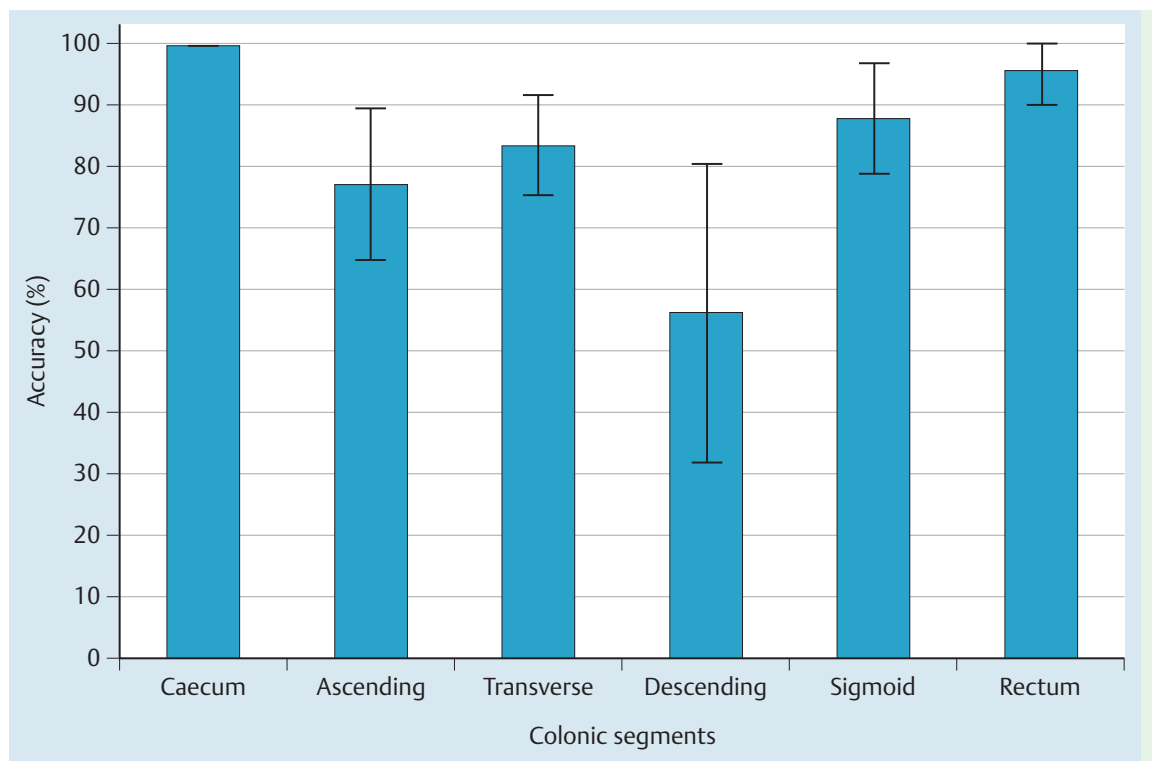

Fig. 2 Accuracy of colonoscopists in each colonic segment $(P<0.001)$.

Our findings are consistent with other retrospective and case studies $[8,21]$. Shah et al showed colonoscopist accuracy at point localization across 100 procedures to be approximately $85 \%$ [22]. They found colonoscopists to be most inaccurate at locating the splenic flexure with only $69 \%$ accuracy. In our data, colonoscopists were most inaccurate in the descending colon. Of the 7 polyps incorrectly localized in the descending colon, 6 were by localized by inexperienced colonoscopists.

There are a number of limitations of our study. Our reference standard for colonoscope tip location (ScopeGuide) may not itself have been accurate, given previous reports of $10 \%$ to $15 \%$ inaccuracy when compared with air contrast enema [14,22]. Also, the relatively small sample size, variation in the number of colonos- copies performed, and the small number of polyps resected by several participating colonoscopists are further limitations. Strengths of our study include the novel assessment of the impact of patient, colonoscopist and procedural variables on accuracy, which have not previously been studied.

In summary, colonoscopists can be inaccurate at localizing lesions during colonoscopy especially within the mid colon. To supplement endoscopic appearances, magnetic endoscope imaging might be a useful tool for the localization of lesions throughout the colon. Further studies are needed to determine how lesion localization at colonoscopy can be improved and other the factors that influence accuracy. 
Table 3 Impact of colonoscopist training and volume on accuracy of polyp localization

\begin{tabular}{|c|c|c|c|c|c|c|}
\hline Colonoscopist & Years since completing training & Annual colonoscopy volume & Total polyps & Correct & Incorrect & Accuracy (\%) \\
\hline 1 & $>10$ & $100-250$ & 6 & 5 & 1 & $83.3 \%$ \\
\hline 2 & $1-5$ & $100-250$ & 36 & 35 & 1 & $97.2 \%$ \\
\hline 3 & $6-10$ & $100-250$ & 5 & 1 & 4 & $20.0 \%$ \\
\hline 4 & 0 & $100-250$ & 31 & 31 & 0 & $100 \%$ \\
\hline 5 & $1-5$ & $250-500$ & 39 & 36 & 3 & $92.3 \%$ \\
\hline 6 & $1-5$ & $>500$ & 45 & 35 & 10 & $77.8 \%$ \\
\hline 7 & $1-5$ & $250-500$ & 15 & 10 & 5 & $66.7 \%$ \\
\hline 8 & $>10$ & $100-250$ & 6 & 5 & 1 & $83.3 \%$ \\
\hline 9 & $6-10$ & $250-500$ & 2 & 2 & 0 & $100 \%$ \\
\hline 10 & $1-5$ & $100-250$ & 3 & 3 & 0 & $100 \%$ \\
\hline 11 & $1-5$ & $100-250$ & 12 & 10 & 2 & $83.3 \%$ \\
\hline 12 & $1-5$ & $100-250$ & 2 & 2 & 0 & $100 \%$ \\
\hline 13 & $1-5$ & $250-500$ & 55 & 45 & 10 & $81.8 \%$ \\
\hline 14 & $1-5$ & $<100$ & 4 & 3 & 1 & $75.0 \%$ \\
\hline
\end{tabular}

\section{Competing interests: None}

\section{Institutions}

${ }^{1}$ Department of Gastroenterology and Hepatology, Princess Alexandra Hospital;

2 School of Medicine, The University of Queensland;

${ }^{3}$ Department of Gastroenterology, Queen Elizabeth II Jubilee Hospital;

${ }^{4}$ Queensland Health Clinical Skills Development Service;

${ }^{5}$ Faculty of Medicine and Biomedical Sciences, \& Faculty of Health and Behavioural Sciences, The University of Queensland; Brisbane, AUSTRALIA

\section{References}

1 Zauber AG, Winawer SJ, O'Brien MJ et al. Colonoscopic polypectomy and long-term prevention of colorectal-cancer deaths. N Engl J Med 2012; 366: $687-696$

2 Frager DH, Frager JD, Wolf EL et al. Problems in the colonoscopic localization of tumors: continued value of the barium enema. Gastrointest Radiol 1987; 12: $343-346$

3 Cho YB, Lee WY, Yun HR et al. Tumor localization for laparoscopic colorectal surgery. World J Surg 2007; 31: 1491 -1495

4 Tabibian N, Michaletz PA, Schwartz JT et al. Use of an endoscopically placed clip can avoid diagnostic errors in colonoscopy. Gastrointest Endosc 1988; 34: $262-264$

5 Piscatelli N, Hyman N, Osler T. Localizing colorectal cancer by colonoscopy. Arch Surg 2005; 140: 932 -935

6 Fletcher RH. The end of barium enemas? N Engl J Med 2000; 342: $1823-1824$

7 Lam DT, Kwong KH, Lam CW et al. How useful is colonoscopy in locating colorectal lesions? Surg Endosc 1998; 12: 839-841

8 Vignati P, Welch JP, Cohen JL. Endoscopic localization of colon cancers. Surg Endosc 1994; 8: 1085-1087

9 Farrar WD, Sawhney MS, Nelson DB et al. Colorectal cancers found after a complete colonoscopy. Clin Gastroenterol Hepatol 2006; 4: 12591264

10 Pabby A, Schoen RE, Weissfeld JL et al. Analysis of colorectal cancer occurrence during surveillance colonoscopy in the Dietary Polyp Prevention Trial. Gastrointest Endosc 2005; 61: 385-391
11 Moss A, Bourke MJ, Pathmanathan N. Safety of colonic tattoo with sterile carbon particle suspension: a proposed guideline with illustrative cases. Gastrointest Endosc 2011; 74: 214-218

12 Jess $P$, Bulut $O$, Almasi $A$ et al. The usefulness of a magnetic endoscope locating device in colonoscopy in daily practice: a prospective casecontrolled study. Surg Endosc 2009; 23: $1353-1355$

13 Ellul P, Fogden E, Simpson C et al. Colonic tumour localization using an endoscope positioning device. Eur J Gastroenterol Hepatol 2011; 23: $488-491$

14 Shah SG, Pearson HJ, Moss S et al. Magnetic endoscope imaging: a new technique for localizing colonic lesions. Endoscopy 2002; 34: 900 - 904

15 von Elm E, Altman DG, Egger $M$ et al. The Strengthening the Reporting of Observational Studies in Epidemiology (STROBE) statement: guidelines for reporting observational studies. Ann Intern Med 2007; 147: $573-577$

16 Leung JW, Thai A, Yen A et al. Magnetic endoscope imaging (ScopeGuide) elucidates the mechanism of action of the pain-alleviating impact of water exchange colonoscopy - attenuation of loop formation. J Interv Gastroenterol 2012; 2: 142 - 146

17 Mark-Christensen A, Brandsborg S, Iversen LH. Magnetic endoscopic imaging as an adjuvant to elective colonoscopy: a systematic review and meta-analysis of randomized controlled trials. Endoscopy 2015; 47: $251-261$

18 Hoff G, Bretthauer M, Dahler S et al. Improvement in caecal intubation rate and pain reduction by using 3-dimensional magnetic imaging for unsedated colonoscopy: a randomized trial of patients referred for colonoscopy. Scand J Gastroenterol 2007; 42: 885-889

19 Nerup N, Preisler L, Svendsen MB et al. Assessment of colonoscopy by use of magnetic endoscopic imaging: design and validation of an automated tool. Gastrointestinal endoscopy 2015; 81: 548-554

20 Cheung HY, Chung CC, Kwok SY et al. Improvement in colonoscopy performance with adjunctive magnetic endoscope imaging: a randomized controlled trial. Endoscopy 2006; 38: 214-217

21 Stanciu C, Trifan A, Khder SA. Accuracy of colonoscopy in localizing colonic cancer. Rev Med Chir Soc Med Nat lasi 2007; 111: 39-43

22 Shah SG, Saunders BP, Brooker JC et al. Magnetic imaging of colonoscopy: an audit of looping, accuracy and ancillary maneuvers. Gastrointest Endosc 2000; 52: $1-8$ 